\title{
Caracterización preliminar de las arenas asfálticas de Picacho, municipio de Pesca (Boyacá, Colombia)
}

\author{
Olga Patricia Gómez-Rojas ${ }^{1 *}$; Ángeles G.-Borrego²; Carlos Perea-Solano"; \\ Oswaldo Alfonso Sánchez-Morales ${ }^{1}$; Hither Fernando Flórez-Molano ${ }^{1}$
}

DOI: http://dx.doi.org/10.18273/revbol.v40n2-2018005 @ ( )

Forma de citar: Gómez-Rojas, O.P., G.-Borrego, A., Perea-Solano, C., Sánchez-Morales, O.A., y FlórezMolano, H.F. (2018). Caracterización preliminar de las arenas asfálticas de Picacho, municipio de Pesca (Boyacá, Colombia). Boletín de Geología, 40(2), 87-99. DOI: 10.18273/revbol.v40n2-2018005.

\section{RESUMEN}

En Colombia las arenas asfálticas las explotan para la pavimentación de vías terciarias, un ejemplo de esto son las areniscas asfálticas de la Formación Picacho, ubicadas en el municipio de Pesca, Boyacá. Este estudio tiene como objetivo una caracterización de las arenas asfálticas con el fin de tener una información preliminar sobre su variación en composición global entre dos frentes de explotación (minas La Emilia y Santa Teresa) con vistas a su posible potencial como fuente de hidrocarburos no convencionales. Los métodos comprenden análisis elementales, físico mecánico, SARA y pirólisis. Los resultados determinan que la unidad está compuesta por areniscas silíceas. Los porcentajes de azufre son bajos en casi todas las muestras, excepto una que alcanza 5\%, la gravedad API determina que los crudos presentan un valor de 22,4 y 9,2 indicando que son crudos medios y extra pesados, las fracciones de crudo reflejan una mayor proporción de resinas y asfáltenos, la pirólisis indica un elevado contenido en volátiles que destila a temperaturas por debajo de $500^{\circ} \mathrm{C}$.

Palabras clave: arenas asfálticas; Formación Picacho; hidrocarburos pesados.

\section{Preliminary bulk characterization of Picacho tar sands, Pesca municipality (Boyacá, Colombia)}

\begin{abstract}
The tar sands are mined in Colombia for paving the local roads. An example of this use is the tar sands of the Picacho Formation, located in the municipality of Pesca, Boyacá. The objective of this study is providing a preliminary bulk characterization to evaluate this resource as a possible source of unconventional hydrocarbons in this area. The study is limited to two fronts of exploitation named the La Emilia and Santa Teresa mines. The methods used comprise elemental analysis, physico-mechanical test, SARA and pyrolysis. The results determine that the unit is composed of siliceous sandstones. The percentages of sulfur are low in most of the samples, except one that reaches 5\%, the API gravity between 22.4 and 9.2 indicates that they range between medium and extra heavy crude, the oil fractional composition reflects a higher proportion of resins and asphaltenes, the pyrolysis indicates a high volatile content that completely distill at temperatures below $500^{\circ} \mathrm{C}$.
\end{abstract}

Keywords: tar sands; Picacho Formation; heavy oil.

\footnotetext{
${ }^{1}$ Escuela de Ingeniería de Minas, Universidad Pedagógica y Tecnológica de Colombia, Colombia. (*)olgapatricia.gomez@uptc.edu.co, carlos.perea@uptc.edu.co,oswaldo.sanchez@uptc.edu.co,fer.floretz@gmail.com ${ }^{2}$ Instituto Nacional del Carbón, Consejo Superior de Investigación Científica CSIC, España. angeles@incar.csic.es
} 


\section{INTRODUCCIÓN}

Los combustibles fósiles representan una gran fuente de energía con la cual se impulsa el desarrollo económico de las naciones desarrolladas y emergentes (ReyesCaballero et al., 2016) y su actual ritmo de vida. Entre los tipos de combustibles fósiles se encuentran las arenas asfálticas o bituminosas, que han sido denominados hidrocarburos fósiles no convencionales.

Estudios enfocados en la caracterización y aprovechamiento han sido reportados en diversos trabajos (Al-Otoom et al., 2010; Hong et al., 2013; Qing et al., 2012; Sherval, 2015; Sleep et al., 2017); algunas investigaciones relacionan el impacto ambiental que genera su utilización (Gerner et al., 2017).

Canadá tiene la tercera mayor reserva de petróleo del mundo, el 95\% de esta ubicada en la provincia de Alberta, siendo el yacimiento de arenas de petróleo de Athabasca la reserva más grande en el mundo (Gerner et al., 2017). Las arenas asfálticas del norte de Alberta,

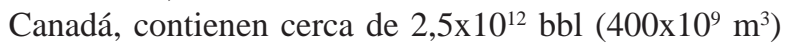
de hidrocarburos pesados llamados bitumen (bitúmenes se pueden definir, desde el punto de vista de explotación, como mezclas viscosas naturales de hidrocarburos de molécula muy pesada). Las reservas iniciales usando las tecnologías actuales de explotación son de cerca de $180 \times 10^{9}$ bbl $\left(30 \times 10^{9} \mathrm{~m}^{3}\right)$ y serían suficientes para satisfacer la demanda interna de petróleo crudo en Canadá por 250 años.

Otros depósitos importantes de arenas asfálticas se encuentran en Arabia Saudita con alrededor de 260×10 ${ }^{9}$ bbl $\left(41 \times 10^{9} \mathrm{~m}^{3}\right)$ y pequeños recursos de estas arenas en Utah (EE.UU.) con 1,8x10 $\mathrm{m}^{3}$ de barriles y un ejemplo cercano se tiene en Venezuela que según US Energy Information Administration (EIA, 2012) contiene miles de millones de barriles de petróleo crudo extra-pesado y depósitos de arenas asfálticas, la mayoría de las cuales están situadas en la Faja del Orinoco en el centro de Venezuela donde las instalaciones tienen capacidad para producir 600 mil barriles por día. En general las arenas asfálticas representan reservas enormes de hidrocarburos; de ahí el interés creciente en este recurso. Colombia, un país con múltiples recursos minero energéticos, en la actualidad cuenta con exploraciones $\mathrm{y}$ explotaciones en diferentes regiones; no obstante algunos no han sido considerados con todo su potencial económico, ya que por ejemplo las areniscas asfálticas o bituminosas presentes en Colombia solo han sido referenciadas en pocos trabajos donde se destaca la localización geográfica y geológica (Jiménez y Cruz,
2004; Sandoval, 2007); al igual que la determinación de las características fisicomecánicas (Ruíz-Acero et al., 2016) que establecen si pueden ser utilizadas en la pavimentación de vías terciarias; en la zona, la pequeña minería que se desarrolla en la explotación no cuenta con mucha información y menos con datos de reservas. De acuerdo con la Agencia Nacional de Hidrocarburos (ANH, 2009) el potencial de reservas de hidrocarburos no convencionales de Colombia es significativo, estudios previos aseguran que las arenas asfálticas en Colombia pueden generar entre 40 - 60 Gbbl (mil millones de barriles) de petróleo recuperable.

En este trabajo no se pretende proporcionar una valoración de las características del yacimiento, ya que se carece de los datos de extensión real y variación en la riqueza de la formación que contiene las arenas asfálticas. Sin embargo, si se pretende proporcionar algunas de las características de las areniscas y de los crudos presentes en la Formación Picacho, con el fin de conocer más acerca de este recurso como posible fuente de hidrocarburos no convencionales mediante el análisis SARA que establece proporciones de compuestos saturados, aromáticos, resinas y asfaltenos, determinación de la composición elemental y pirólisis, análisis comunes cuando se tiene el propósito de determinar la riqueza de las arenas y la variación de unos puntos a otros de muestreo.

\section{GEOLOGÍA DEL YACIMIENTO}

La Formación Picacho de edad Eoceno inferior fue denominada como un conjunto de areniscas que forman el Cerro Picacho en el alto de El Portillo, al noroeste del casco urbano de Paz del Río. Litológicamente esta unidad se caracteriza por presentar areniscas de color blanco de grano grueso a fino, alta porosidad y permeabilidad, las cuales se encuentran impregnadas de hidrocarburos, el origen de esta Formación es continental, fue depositada en un ambiente posiblemente de tipo deltaico, de gran extensión lateral (Reyes, 1984). La Formación Picacho equivale a las areniscas de Lenguazaque de Van der Hammen (1957, tomado de Reyes, 1984), que representa el nivel arenoso inferior de la Formación Bogotá y se correlaciona bien con la Formación Mirador de Norte de Santander.

El estudio se enfoca particularmente en dos minas de la zona, las minas Santa Teresa y La Emilia en las cuales encontramos un gran paquete de areniscas impregnadas que actualmente se explotan como material asfáltico, sin que se tenga una estimación de sus reservas y características, debido a la forma artesanal de su explotación. 
El municipio de Pesca, Boyacá (FIGURA 1), se encuentra ubicado en el flanco Este de la Cordillera Oriental, limita al norte con Firavitova, al sur con Zetaquira, por el oriente con Iza, Cuitiva y Tota y por el occidente con Rondón, Siachoque y Toca. El sector está comprendido en la plancha 191 - II - D - 1 del Instituto Geográfico Agustín Codazzi (IGAC).

La cabecera municipal está localizada a los 5³4' de Latitud Norte y $73^{\circ} 03^{\prime} \mathrm{W}$ a una altura de 2631 m.s.n.m. con temperaturas promedio de $14^{\circ} \mathrm{C}$ propios de clima frio. El yacimiento de la mina Santa Teresa está delimitado por una vía carreteable adyacente que sigue la dirección del río Pesca con rumbo N14º W y según el sistema de coordenadas planas del IGAC con origen en Bogotá, el área de estudio se enmarca dentro de las siguientes coordenadas en $\mathrm{X}$ : 1'106.890 y en Y: 1’113.550 a una cota de 2599,24 m.s.n.m. La mina La Emilia se encuentra hacia el NE del municipio de Pesca, entre las coordenadas geográficas X: 1'109.000 a X: 1'110.000; Y: 1'117.000 a Y: 1'118.000 entre las quebradas El Mode y Cristalal (FIGURA 1).

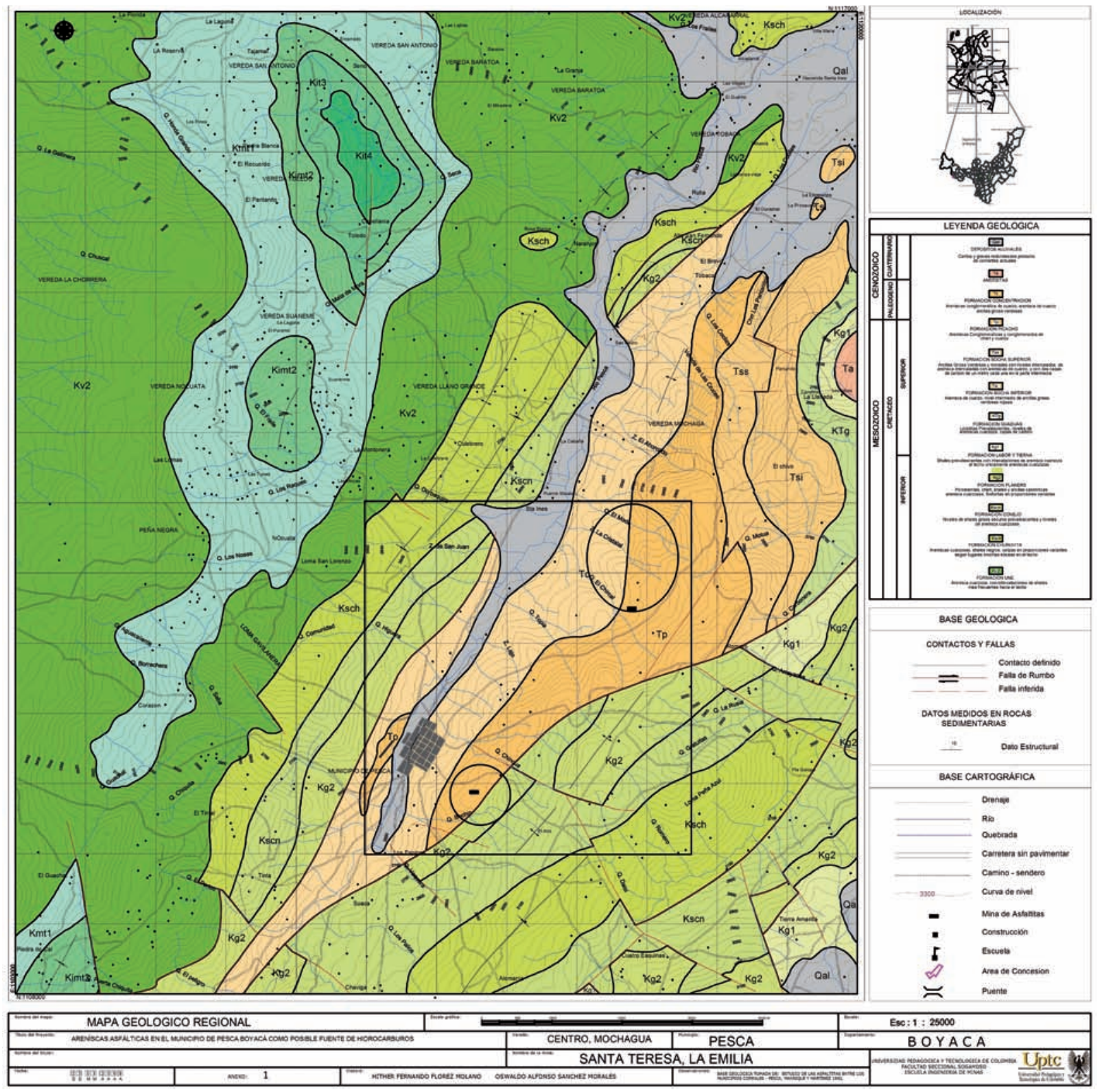

FIGURA 1. Localización y mapa geológico del área de estudio. Modificada plancha 191 - II - D del IGAC. Escala 1:25.000. 


\section{METODOLOGÍA}

En lugares frescos, se tomaron nueve muestras de las cuales ocho corresponden a arenas impregnadas de aceite; estas se tomaron aleatoriamente abarcando diversos niveles de los frentes de explotación de la mina Santa Teresa (P1, P2, P3, P4, P5) actualmente en explotación y de la mina La Emilia (P6, P7 y P8), y una de crudo líquido (codificada B1) del techo de la mina Santa Teresa; las muestras de areniscas asfálticas fueron preparadas con el fin de extraer el crudo para su análisis (estos crudos extraídos se codifican para las minas La Emilia y Santa Teresa respectivamente con Q1 y Q2) y se hizo la caracterización de las arenas de la unidad mediante petrografía.

En la etapa de preparación se realizaron varias pruebas de separación recomendadas en la literatura como se describen a continuación. Para la primera prueba de recuperación del bitumen se utilizó agua caliente a $100^{\circ} \mathrm{C}$, temperatura baja que no altera sus propiedades (HWEP, The Hot Water Extraction Process) técnica reportada en los trabajos de Liu et al. (2006) y Sparks et al. (2003) con poca recuperación. Finalmente se realizó la extracción con éter etílico con lo que se logró la separación efectiva, y recuperar el disolvente, pero tiene el inconveniente de ser un reactivo de venta restringida en Colombia.

Los siguientes análisis se realizaron a las muestras, algunas pruebas fueron realizadas a las muestras tal como fueron recolectadas (P1 - P9) y al crudo (B1). Dos muestras con código Q1 y Q2 se refieren a las muestras de crudo de la mina La Emilia y la mina Santa Teresa extraídas con éter etílico, respectivamente.

El análisis petrográfico se realizó a ocho (8) muestras de areniscas una vez fueron separadas de los hidrocarburos. Se analizaron utilizando microscopía óptica de luz transmitida, se identificaron y cuantificaron minerales y sus características texturales.

La pirólisis es en esencia un calentamiento en atmósfera inerte. En el caso de las rocas orgánicas este proceso se utiliza como método de caracterización, para estudiar la temperatura a la que destilan diversos componentes que se encuentran en la roca en estado sólido. Entre algunos de los ensayos bien conocidos de pirólisis que se utilizan para la caracterización de rocas madre, figura el ensayo Rock-Eval, que proporciona información sobre el potencial generador de aceites entre diversas temperaturas de corte utilizando una rampa de calentamiento de $25^{\circ} \mathrm{C} / \mathrm{min}$ hasta $600^{\circ} \mathrm{C}$
(Espitalié et al., 1977), llegando el Rock-Eval 6 hasta los $850^{\circ} \mathrm{C}$ e incorporando la posibilidad de variar la rampa de calentamiento. Para la caracterización del rendimiento en aceite de la lutita aceitosa se utiliza habitualmente el ensayo Fischer que emplea una temperatura máxima de $500^{\circ} \mathrm{C}$ y una rampa de calentamiento de $12^{\circ} \mathrm{C} / \mathrm{min}$ (ASTM D 3904). En general las atmósferas que se utilizan para el ensayo suelen ser de helio o nitrógeno y la velocidad de calentamiento puede variarse en función del detalle que se quiera alcanzar para identificar los diversos procesos entre pocos grados y unas decenas de grados por minuto. Cuanto más lenta sea la velocidad de calentamiento, mejor se separarán los procesos, lo que permitirá un estudio más detallado del material problema. Si el equipo permite el registro de la pérdida de masa con el aumento de la temperatura, la primera derivada de esta curva constituye un pirograma en el que se pueden observar los diversos procesos de pérdida de masa, la temperatura a la que ocurren, su intensidad y la rapidez con la que suceden, todo ello información importante si se quiere realizar un aprovechamiento de fracciones destiladas de un bitumen pesado. En un pirograma de un componente orgánico hay una pérdida de masa en torno a $100^{\circ} \mathrm{C}$ debido a la humedad, a continuación una pérdida de masa debido a volátiles atrapados, seguida de la correspondiente a la rotura térmica de la materia orgánica, bien sea kerógeno o bitúmenes más o menos pesados, para terminar con las pérdidas debidas a condensación del residuo carbonoso (Lechner et al., 1989). Ya que muchos de los procesos de recuperación de los bitúmenes de las arenas llevan involucrado calentamiento térmico (Speight, 2016)se consideró interesante realizar un estudio del comportamiento en pirólisis de las arenas con el fin de estudiar las temperaturas relevantes a las que ocurren los principales procesos de descomposición térmica. Para poder separar los procesos con nitidez se eligió una baja velocidad de calentamiento $\left(5^{\circ} \mathrm{C} / \mathrm{min}\right)$ y una temperatura máxima elevada $\left(815^{\circ} \mathrm{C}\right)$ que garantice que todos los procesos de descomposición química serán observables.

Los ensayos de pirolisis se llevaron a cabo calentando la muestra hasta $815^{\circ} \mathrm{C}$ a $5^{\circ} \mathrm{C} / \mathrm{min}$ en flujo de nitrógeno de $35 \mathrm{~mL} / \mathrm{min}$. Se eligió $815^{\circ} \mathrm{C}$ porque es la temperatura a la que se determinan las materias volátiles de los combustibles fósiles y una velocidad de calentamiento lenta para poder diferenciar los procesos que tienen lugar durante el calentamiento. Una vez que la masa a $815^{\circ} \mathrm{C}$ es constante se cambia el flujo a aire para realizar la combustión del coque formado y obtener el contenido en cenizas. De esta manera se obtienen una 
serie de datos como contenido en volátiles, cenizas y humedad que son similares al análisis inmediato.

Los análisis fisicoquímicos del asfalto se realizaron en el Laboratorio de Crudos y Derivados en la Universidad Nacional de Colombia, Sede Medellín, a los crudos Q1 y Q2, se realizó el método de prueba estándar para agua en productos de petróleo y materiales bituminosos por destilación bajo la norma ASTM D 95, agua y sedimentos (ASTM D 96), método de prueba estándar para cenizas en pro del petróleo (ASTM D 482), método de prueba estándar para azufre en productos del petróleo (ASTM D 129), método de prueba estándar para gravedad API de petróleo crudo y productos del petróleo (ASTM D 287), método de prueba estándar para calor de combustión de combustibles hidrocarburos líquidos por bomba calorimétrica (ASTM D 240), determinación de residuos de carbón que queda después de la evaporación y pirólisis de un aceite, prueba Conradson del productos del petróleo (ASTM D 189) y método de prueba estándar para viscosidad Saybolt (ASTM D 88).

Los análisis SARA cuantifican varias fracciones de compuestos con diferente polaridad: compuestos saturados (hidrocarburos $n$-alcanos, ramificados y saturados cíclicos), aromáticos (compuestos con uno o varios anillos aromáticos), resinas y asfaltenos (ambos con sustituyentes polares, siendo los asfaltenos insolubles en heptano o pentano y las resinas solubles. Estos análisis se realizaron a las muestras liquidas extraídas de la mina La Emilia y de la mina Santa Teresa en el laboratorio de ensayos para asfaltos y mezclas asfálticas de CORASFALTOS. El procedimiento utilizado fue cromatografía en capa delgada (TLC), que no requiere separación previa de los asfaltenos y emplea detector FID (Flame Ionization Detector) para la cuantificación. Los disolventes utilizados como eluyentes fueron n-hexano, tolueno y diclorometano/metanol. Este procedimiento es adecuado para el análisis de crudos de alto peso molecular y compleja estructura o composición química como es el caso de la evaluación de crudos pesados o precursores para la producción de asfaltos refinados.

Los análisis elemental e inmediato se realizaron a los crudos Q1, Q2 y B1 con el fin de determinar el contenido de materia volátil, cenizas, rendimiento de coque, contenido de carbono y azufre, esto permite evaluar las características del combustible y de los residuos que generan su refinamiento y utilización.

\section{RESULTADOS Y DISCUSIÓN}

\section{Caracterización petrográfica}

La Formación Picacho aflora hacia el margen izquierdo del río Pesca y su composición litológica la conforman predominantes paquetes de areniscas con alta porosidad y permeabilidad las cuales almacenan asfalto natural o bitumen, estas rocas son maduras mineralógicamente y alcanzan hasta $6 \mathrm{~m}$ de espesor, seguidas de un grueso paquete de arcillas grises a rosadas con intercalaciones de arenisca de grano fino.

De acuerdo con la clasificación de Pettijohn et al. (1987), estas rocas de la Formación Picacho se clasifican en areniscas cuarzosas de grano medio a grueso, areniscas conglomeráticas y conglomerados arenosos, compuestos principalmente por cuarzo entre 95 y $84 \%$; fragmentos de metacuarcita tamaño arena entre 40 y $4 \%$; porcelanita entre 16 y 5\% y pedernal que alcanza hasta un porcentaje de $7 \%$ en las diferentes muestras y en menor proporción (menos del 5\%) arcillas (FIGURA 2).

\section{Análisis elemental e inmediato}

Los resultados se pueden observar en la TABLA 1. Como resultado de los procesos de alteración (biodegradación, oxidación, etc.) los hidrocarburos pesados tienen altas concentraciones en sulfuros; como se observa los porcentajes de azufre oscilan entre $1,7 \%$ y $5 \%$.

Las muestras estudiadas varían en contenido de cenizas de 85 a $92 \%$ y el contenido de C de 5 a $12 \%$. El contenido de azufre fue bajo para todas las muestras, pero alcanzó valores superiores al $2 \%$ en los alquitranes. Solo dos muestras produjeron un contenido de azufre significativamente mayor. La relación atómica H/C indica un alto potencial de hidrocarburos que tiende a aumentar del piso al techo del yacimiento, dando variabilidad vertical al yacimiento. El valor calorífico del alquitrán libre fue $7076 \mathrm{kcal} / \mathrm{g}$, pero para las arenas de alquitrán disminuyen alrededor de 700 $\mathrm{kcal} / \mathrm{g}$. El rendimiento en coque (residuo carbonoso generado tras la destilación a $815^{\circ} \mathrm{C}$ ) es muy bajo en todas las muestras y el cociente entre materia volátil y rendimiento en coque oscila entre 12,7 y 18,7 indicando que la mayor parte del material de las arenas asfálticas es destilable. 

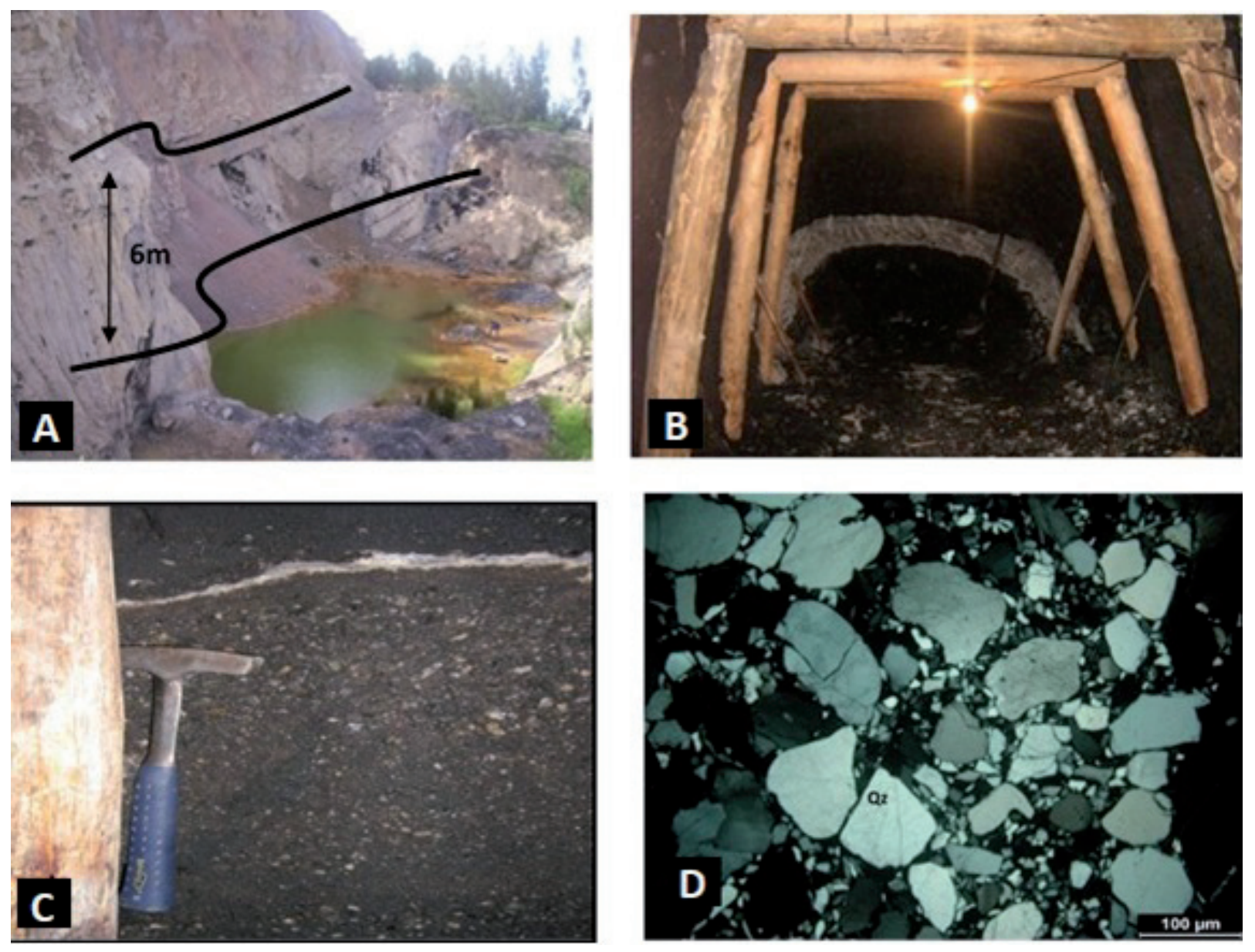

FIGURA 2. Areniscas asfálticas de la mina Santa Teresa. A-C. Lugares donde fueron extraídas algunas muestras. D. Microfotografía en luz transmitida de las areniscas de la Formación Picacho, se observa en la figura un alto contenido de sílice, alcanzando hasta un 95\% de cuarzo sub redondeado y menor proporción de arcilla.

TABLA 1. Resultados de análisis elemental e inmediato. Abreviaciones: Cz: cenizas; MV: Materia volátil; RC: Rendimiento coque; C: carbono; S: azufre; bs: base seca; slcz: base seca libre de cenizas. B1 muestra líquida, P1 - P8 arenas asfálticas.

\begin{tabular}{|c|c|c|c|c|c|c|c|c|}
\hline Muestra & $\begin{array}{c}\% \mathrm{Cz} \\
\text { [bs] }\end{array}$ & $\begin{array}{c}\text { \%MV } \\
\text { [bs] }\end{array}$ & $\begin{array}{c}\text { \% RC } \\
\text { [bs] }\end{array}$ & $\begin{array}{l}\% C \\
\text { [bs] }\end{array}$ & $\begin{array}{l}\% S \\
\text { [bs] }\end{array}$ & $\begin{array}{c}\% \mathrm{~S} \\
\text { [slcz] }\end{array}$ & $\begin{array}{c}\text { Relación } \\
\text { atómica } \\
\text { H/C }\end{array}$ & $\begin{array}{c}\text { Relación } \\
\text { atómica } \\
\text { S/C }\end{array}$ \\
\hline B1 & & 94,7 & 2,1 & 65,1 & 1,67 & 1,7 & 2,06 & 0,010 \\
\hline $\mathrm{P} 1$ & 91,0 & 6,5 & 0,4 & 5,9 & 0,19 & 2,1 & 1,79 & 0,012 \\
\hline P2 & 86,9 & 11,5 & 0,7 & 11,6 & 0,28 & 2,1 & 1,79 & 0,009 \\
\hline P3 & 88,9 & 18,7 & 1,2 & 7,3 & 0,56 & 5,0 & 1,79 & 0,029 \\
\hline P4 & 85,4 & 12,3 & 0,8 & 12,7 & 0,31 & 2,1 & 1,66 & 0,009 \\
\hline P5 & 87,8 & 10,5 & 0,4 & 9,3 & 0,31 & 2,5 & 1,81 & 0,012 \\
\hline P6 & 88,5 & 12,3 & 1,0 & 8,5 & 0,32 & 2,8 & 1,67 & 0,014 \\
\hline P7 & 88,1 & 8,5 & 0,1 & 8,0 & 0,30 & 2,5 & 2,26 & 0,014 \\
\hline P8 & 92,6 & 7,5 & 0,4 & 5,2 & 0,30 & 4,1 & 2,94 & 0,022 \\
\hline
\end{tabular}




\section{Ensayos de pirólisis}

Las muestras presentaron un comportamiento complejo con al menos dos picos que se diferencian claramente. La mayoría tiene un perfil similar a la de P2 (FIGURA 3) con un amplio pico cuyo máximo se sitúa a temperaturas entre 210 y $270^{\circ} \mathrm{C}$ y un pico bien marcado alrededor de los $430^{\circ} \mathrm{C}$. Estos picos corresponden a la destilación de compuestos enlazados de diferente forma (FIGURA 3).

Dos muestras presentan perfiles particularmente complejos, la muestra B1, con un pico adicional a los $120^{\circ} \mathrm{C}$, que en principio no sería atribuible a la presencia de agua, ya que la pérdida de masa tiene lugar a temperaturas superiores a $100^{\circ} \mathrm{C}$ y la muestra P3, que además del pico antes mencionado, tiene una pérdida de masa alrededor de $495^{\circ} \mathrm{C}$.

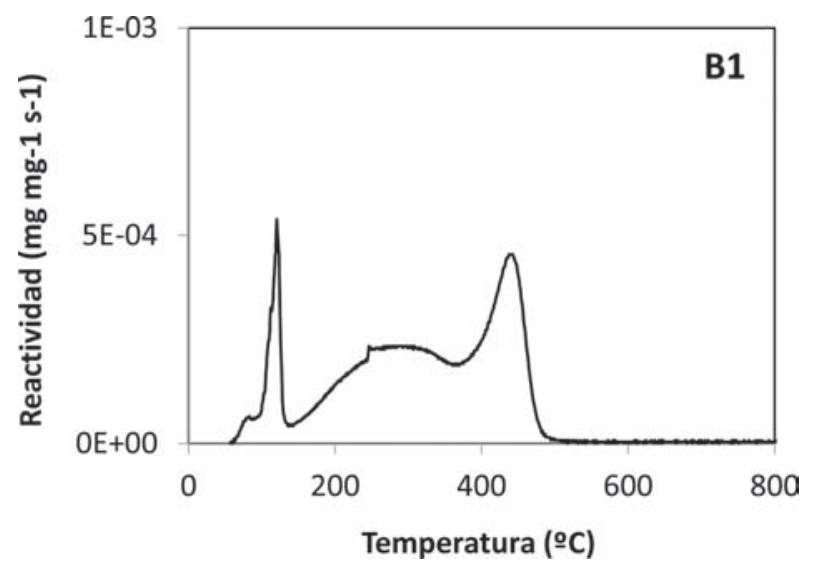

Se realizó un secado en estufa de vacío a $50^{\circ} \mathrm{C}$ y a $150^{\circ} \mathrm{C}$ durante una hora. Ambas temperaturas se consideran lo bastante bajas como para alterar mínimamente la muestra. En los termogramas de las muestras calentadas (FIGURA 4) se observa una disminución muy importante del pico a $120^{\circ} \mathrm{C}$ cuando la muestra es calentada a $50^{\circ} \mathrm{C}$ y la desaparición de este pico a $150^{\circ} \mathrm{C}$ en la muestra B1. En la muestra P3, una temperatura de $50^{\circ} \mathrm{C}$ es suficiente para eliminar el pico a $120^{\circ} \mathrm{C}$. No se observa que varíe la intensidad ni la temperatura a la que se producen los otros picos de destilación indicando una mínima alteración de la muestra. El hecho de que la banda de $120^{\circ} \mathrm{C}$ desaparezca con el secado a vacío a baja temperatura indica que, aunque esta banda no es agua libre, si está relacionada con la humedad de la muestra. En la muestra P3 al calentar a vacío disminuye de forma muy importante el pico a $495^{\circ} \mathrm{C}$.

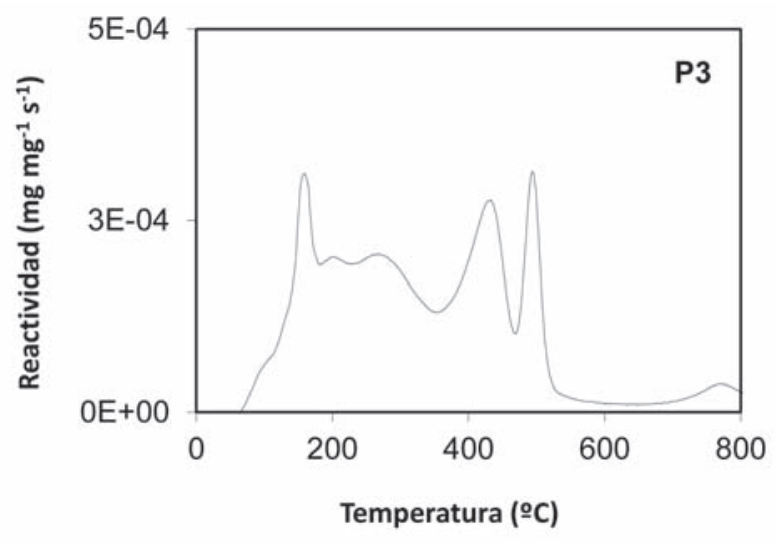

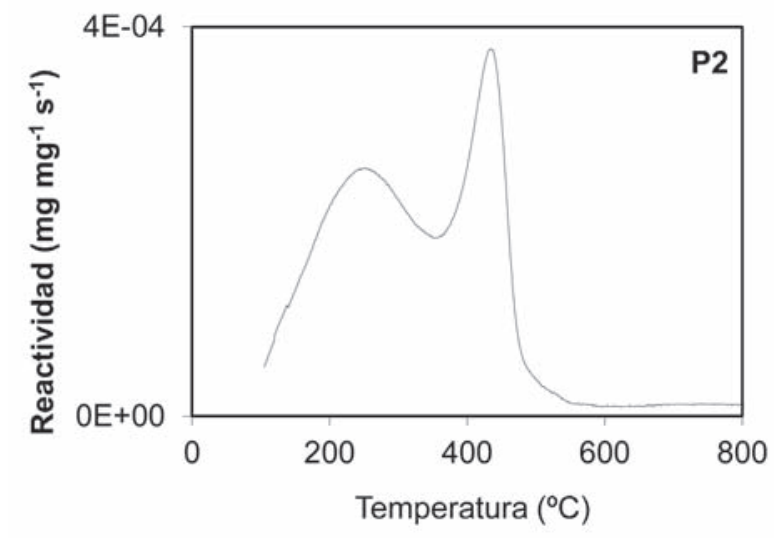

FIGURA 3. Resultados de la pirolisis (B1, P2, P3) de las muestras de petróleo extraídas de la Formación Picacho, Boyacá. 

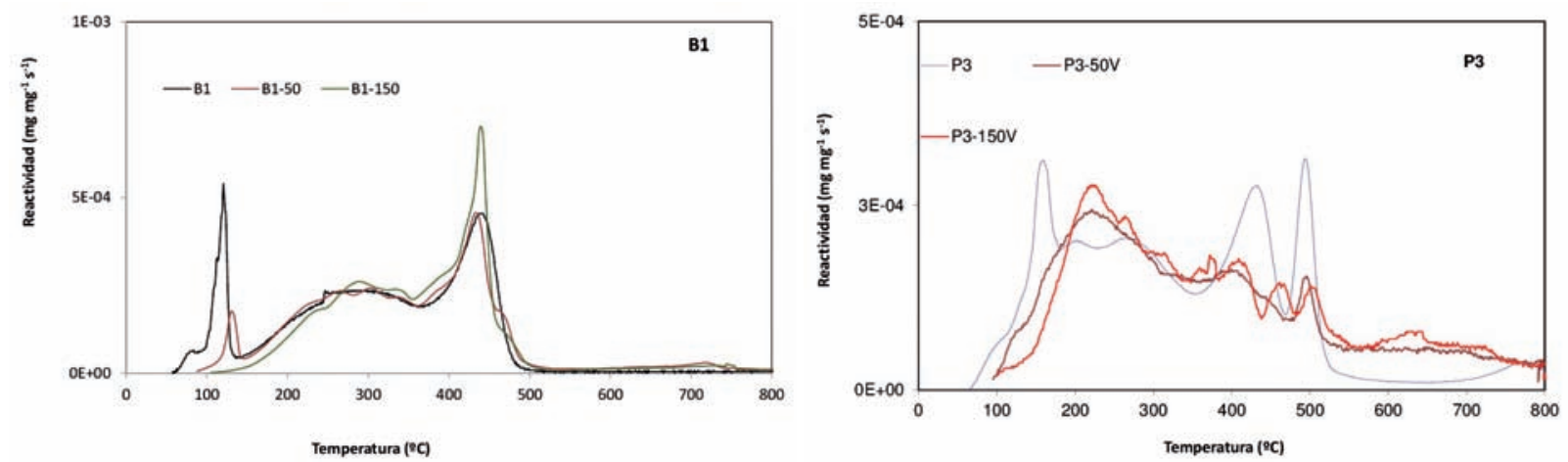

FIGURA 4. Resultados de la pirolisis de las muestras de petróleo extraídas de la Formación Picacho (B1, P3) antes y después del secado al vacío a 50 y $150^{\circ} \mathrm{C}$ durante una hora.

La pérdida de total de peso se completa a temperaturas por debajo de $\operatorname{los} 600^{\circ} \mathrm{C}$ y en la mayoría de las muestras por debajo de $500^{\circ} \mathrm{C}$. La FIGURA 5 muestra la variación de las reactividades de los dos picos principales en las diferentes muestras. La reactividad del pico 1 es en general menor que la del pico 2 excepto en las muestras P7 y P8 cuyas reactividades en ambos picos son prácticamente iguales. Además, el pico 1 muestra una reactividad constante, que solo es ligeramente menor en la muestra $\mathrm{P} 3$. La reactividad del pico 2 presenta una mayor variación y el valor más elevado corresponde al bitumen.

Se pueden establecer algunas diferencias entre las muestras en función de algunas características de los dos picos principales de pirólisis. En general el cociente

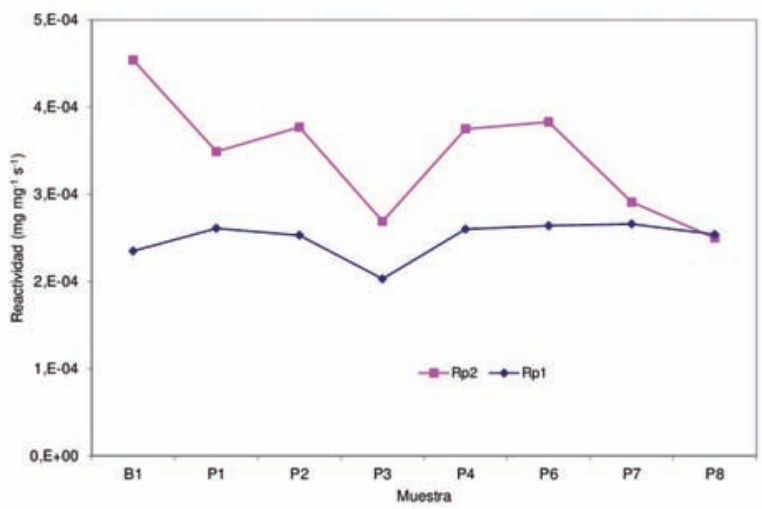

FIGURA 5. Variación de la reactividad de los dos picos principales observados en la pirólisis de las arenas asfálticas de Boyacá. del área entre los dos picos (Ap2/Ap1) se mueve entre 1,30 y 1,38 con la excepción de B1 y P8 que tienen una relación algo menor y el valor mayor observado para P3 debido probablemente a la división del segundo pico en 2. El cociente entre el rendimiento en volátiles y en coque disminuye al aumentar la relación atómica H/C pero la correlación es muy pobre. También se ha observado una relación lineal entre el cociente de las reactividades del pico 2 y pico 1 (FIGURA 6) con la relación atómica H/C.

La reactividad del coque generado durante la pirólisis fue similar en todas las muestras, excepto en la muestra de crudo (B1) y en la P8 que presentaron reactividades más altas, coincidiendo con las muestras que tenían una menor relación Ap2/Ap1.

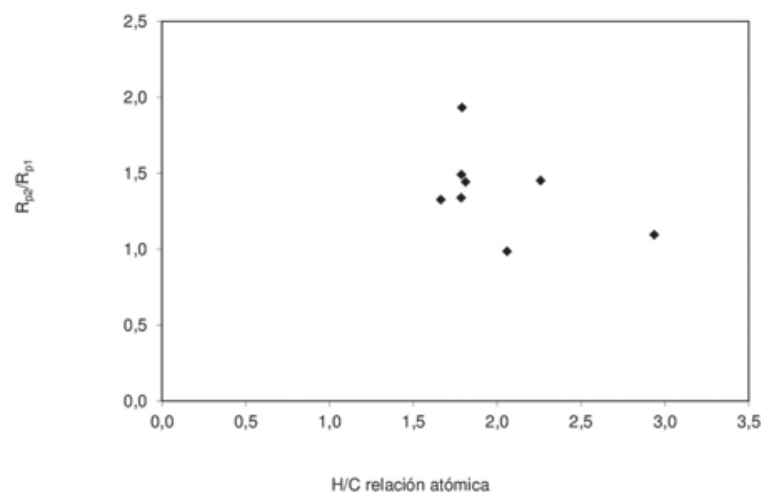

FIGURA 6. Relación entre dos picos principales de pirolisis y la relación atómica H/C. 


\section{Análisis fisicoquímicos}

Los resultados de los análisis fisicoquímicos se relacionan a continuación en la TABLA 2. La gravedad
API medida a los crudos extraídos alcanza valores de 22,4 en Q1 y de 9,2 en Q2, indicando que el tipo de crudo es mediano y extra pesado respectivamente.

TABLA 2. Análisis fisicoquímicos de dos crudos extraídos de las arenas asfálticas de la Formación Picacho, Boyacá. SUS: Segundo Saybolt Universal, Q1 y Q2. Crudo extraído mina La Emilia y mina Santa Teresa respectivamente.

\begin{tabular}{cccc}
\hline ANÁLISIS & UNIDADES & Q1 & Q2 \\
\hline Agua por Destilación & \% Vol. & 0 & 0,4 \\
Agua y Sedimentos & $\%$ Vol. & 0,2 & 0,3 \\
Cenizas & $\% \mathrm{P} / \mathrm{P}$ & 1,8 & 6,3 \\
Contenido de Azufre & $\% \mathrm{P} / \mathrm{P}$ & 1,6 & 1,9 \\
Gravedad API a $60^{\circ} \mathrm{F}\left(15,56^{\circ} \mathrm{C}\right)$ & $\mathrm{N} / \mathrm{A}$ & 22,4 & 9,2 \\
Poder Calorífico & $\mathrm{BTU} / \mathrm{Lb}$ & 16,608 & 16,881 \\
& $(\mathrm{~kJ} / \mathrm{kg})$ & -38630 & -39265 \\
Residuo Carbonoso Conradson & $\% \mathrm{P} / \mathrm{P}$ & 12,5 & 14,4 \\
Viscosidad Saybolt & SUS & 225 & 345 \\
\hline
\end{tabular}

\section{Composición Química del Asfalto, SARA}

La industria del petróleo separa el crudo en cuatro fracciones que son saturados, aromáticos, resinas y asfaltenos. Los resultados de la determinación de las fracciones de los diferentes tipos de hidrocarburos, se reportan en TABLA 3. Los porcentajes de hidrocarburos pesados como resinas y asfaltenos se encuentran en mayores proporciones sumando más de 60\%, con predominancia clara de las resinas. Se puede observar en la TABLA 4 las características de algunos crudos de algunas reservas de arenas asfálticas lo que permite comparar estos yacimientos; cabe destacar que la mina Santa Teresa presenta una gravedad ${ }^{\circ}$ API muy parecida al de Atabasca, Canadá y por el contrario la mina La Emilia se asemeja al de Brasil y Estados Unidos, pero a diferencia de todos como se mencionó anteriormente el contenido en resinas es dos y tres veces mayor.
Entre las dos últimas fracciones SARA, resinas y asfaltenos, no existe una barrera definida, pues ambas reúnen compuestos bastante polares, de alto peso molecular, además de contener anillos aromáticos condensados, heteroátomos y cadenas alquílicas (Alayon, 2004). La presencia de altos contenidos de asfáltenos y resinas en un crudo de acuerdo a Hunt (1995) se puede dar debido a tres causas: cuando el crudo es inmaduro, se dan mayor contenido de asfáltenos y resinas; por biodegradación de la materia orgánica, ya que las bacterias prefieren los hidrocarburos livianos, saturados y aromáticos y por migración de los hidrocarburos livianos, quedando los pesados. Los porcentajes de resinas en los crudos mayores a $50 \%$ indicarían algunas de estas causas, se deben realizar una caracterización mayor que permita conocer la roca fuente.

TABLA 3. Resultados del análisis SARA de los crudos extraídos de las arenas asfálticas de la Formación Picacho, Boyacá. Q1 y Q2. Crudo extraído mina La Emilia y mina Santa Teresa respectivamente

\begin{tabular}{cccccc}
\hline \multicolumn{5}{c}{ FRACCIÓN (\%) } & \\
\hline MUESTRA & Saturados & Aromáticos & Resinas & Asfáltenos & $\begin{array}{c}\text { Asfáltenos/ } \\
\text { resinas }\end{array}$ \\
\hline Q1 & $18,05+/-1,28$ & $22,97+/-2,28$ & $51,90+/-1,30$ & $7,07+/-1,10$ & 0,14 \\
Q2 & $15,22+/-1,28$ & $22,70+/-1,30$ & $54,13+/-1,30$ & $7,95+/-1,10$ & 0,14 \\
\hline
\end{tabular}


TABLA 4. Resinas y asfaltenos de diferentes crudos. Modificado de Demirbas (2002).

\begin{tabular}{lcccc}
\hline \multicolumn{1}{c}{ País } & ${ }^{\circ}$ API & $\begin{array}{c}\text { Resinas } \\
\text { (wt. } \%)\end{array}$ & $\begin{array}{c}\text { Asfaltenos } \\
\text { (wt.\%) }\end{array}$ & $\begin{array}{c}\text { Asfaltenos/ } \\
\text { resinas }\end{array}$ \\
\hline Canadá, Atabasca & 8,3 & 14,0 & 15,0 & 1,07 \\
Venezuela, Boscan & 10,2 & 29,4 & 17,2 & 0,58 \\
Canadá, Cold Lake & 10,2 & 25,0 & 13,0 & 0,52 \\
México, Panucon & 11,7 & 26,0 & 12,5 & 0,48 \\
USA, MS, Baxterville & 16,0 & 8,9 & 17,2 & 1,93 \\
Rusia, Kaluga & 16,7 & 20,0 & 0,5 & 0,03 \\
USA, TX, Hould & 19,7 & 12,0 & 0,5 & 0,04 \\
Brasil, Campos, Atabasca & 19,7 & 21,6 & 2,8 & 0,13 \\
USA, CA, Huntington Beach & 26,2 & 19,0 & 4,0 & 0,21 \\
Canadá, Alberta & 29,0 & 8,5 & 5,3 & 0,62 \\
USA, LA, Brookhaven & 30,6 & 4,6 & 1,7 & 0,36 \\
Rusia, Balachany & 31,7 & 6,0 & 0,5 & 0,08 \\
Rusia, Bibi-Eibat & 32,1 & 9,0 & 0,3 & 0,03 \\
Rusia, Dossor & 32,6 & 2,5 & 0,0 & 0,00 \\
Rusia, Surachany & 35,0 & 4,0 & 0,0 & 0,00 \\
USA, TX, Mexia & 36,0 & 5,0 & 1,3 & 0,26 \\
Iraq, Kirkuk & 36,1 & 15,5 & 1,3 & 0,08 \\
México, Tecoaminocan & 36,7 & 8,8 & 1,5 & 0,17 \\
Colombia, Mina La Emilia** & 22,4 & 51,9 & 7,07 & 0,14 \\
Colombia, Mina Santa Teresa** & 9,2 & 54,13 & 7,95 & 0,14 \\
\hline
\end{tabular}

**Autores

Los resultados del análisis elemental de los crudos extraídos con éter etílico de las minas La Emilia y Santa Teresa, se observan en la TABLA 5.

TABLA 5. Resultados ensayos elementales de crudos Q1 y Q2. Crudo extraído mina La Emilia y mina Santa Teresa respectivamente, B1 muestra liquida.

\begin{tabular}{lccc}
\hline \multicolumn{1}{c}{ Análisis (\%) } & Q1 & Q2 & B1 \\
\hline Carbono & 74,44 & 67,70 & 71,24 \\
Hidrógeno & 10,23 & 9,13 & 10,64 \\
Nitrógeno & 0,41 & 0,48 & 0,39 \\
Oxígeno & 15,9 & 22,69 & 17,73 \\
\hline
\end{tabular}

Los resultados de la caracterización de los crudos presentes en estas arenas asfálticas de la Formación Picacho, comparadas con resultados de la literatura de otras areniscas asfálticas (TABLA 4), muestran que su gravedad API de 22,4 y 9,2 se encuentran dentro de los valores reportados en las de Atabasca, Canadá, de 8,3 (extra pesado) y Alberta, Canadá, 29,0 (crudo mediano), aunque las proporciones de resinas y asfaltenos son diferentes (Demirbaş, 2002) y muestran pocas variaciones entre ellas. La baja proporción de saturados y aromáticos en los asfaltos de la Formación Picacho que son las fracciones más aprovechables para la generación de crudos ligeros a partir de asfaltos pesados y la elevada proporción de resinas, muy por encima de las observadas en bitúmenes de diversas localizaciones en el mundo limitarán de forma muy importante sus posibilidades de utilización.

El estudio de la distribución de las fracciones de SARA en Athabasca les permitió identificar que dependía de la termodinámica y era susceptible de tratamiento térmico; observaron que destilación a temperatura 298 a 398 K de las fracciones más ligeras podría enriquecerlas en 
saturados y aromáticos en la parte externa reduciendo la proporción de resinas (He et al., 2013) lo que podría mejorar sus posibilidades de aprovechamiento como fuente de hidrocarburos convencionales.

\section{CONCLUSIONES}

Los resultados preliminares del estudio de las arenas asfálticas de la Formación Picacho en Pesca, indican una moderada variabilidad de impregnación del crudo en los distintos sitios de muestreo, el alquitrán contenido tiene un $2 \%$ de azufre promedio, es de alto valor calórico, por su alta proporción H/C y alta conversión a volátiles.

La gravedad API determina que los crudos presentes en esta unidad son medio y extra pesado $(22,4$ y 9,2$)$. Su viscosidad es muy elevada, por lo que no pueden ser recuperados por medios tradicionales (bombeo). Desde el punto de vista geoquímico, la fracción dominante es la de resinas que se encuentra en proporción muy superior a la de la mayoría de bitúmenes asfalticos descritos en el mundo.

La baja proporción de saturados y la elevada proporción de resinas limitan las posibilidades de explotación de las arenas de la Formación Picacho como fuente no convencional de hidrocarburos, aunque estos estudios son preliminares, falta realizar más análisis, mayor número de muestras y cálculos de reservas los cuales permitirán una caracterización más detallada de los compuestos presentes en los bitúmenes.

Los resultados indican que por debajo de $500^{\circ} \mathrm{C}$ se craquean la mayor parte de los bitúmenes liberándose hidrocarburos más ligeros, y unos $320^{\circ} \mathrm{C}$ serían suficientes para craquear el $60 \%$ de los volátiles de las arenas asfálticas de Pesca. Esto indica que a una temperatura relativamente baja se puede obtener un aceite más ligero que simplificaría posteriores tareas de refino.

\section{AGRADECIMIENTOS}

Los autores expresan sincero agradecimiento a la Dirección de Investigaciones de la Universidad Pedagógica y Tecnológica de Colombia DIN- CIFAS y al Consejo Superior de Investigación Científica CSIC y al Instituto Nacional del Carbón INCAR, Oviedo, España.

\section{REFERENCIAS}

Al-Otoom, A., Allawzi, M., Al-Omari, N., and AlHsienat, E. (2010). Bitumen recovery from Jordanian oil sand by froth flotation using petroleum cycles oil cuts. Energy, 35(10), 42174225. doi: 10.1016/j.energy.2010.07.008.

Alayon, M. (2004). Asfaltenos. Ocurrencia y floculación. Cuadernos FIRP. Mérida: Universidad de los Andes.

ANH. (2009). Petróleo y futuro. Agencia Nacional de Hidrocarburos. Consultado el 20 de noviembre de 2014. http://censat.org/es/analisis/loshidrocarburos-no-convencionales-un-crudopanorama-2.

ASTM D 88. Standard Test Method for Saybolt Viscosity. American Society for Testing and Materials (ASTM). 2013.

ASTM D 95. Standard Test Method for Water in Petroleum Products and Bituminous Materials by Distillation. American Society for Testing and Materials (ASTM). 2005.

ASTM D 96. Standard Test Methods for Water and Sediment in Crude Oil by Centrifuge Method (Field Procedure). ASTM D96-88. American Society for Testing and Materials (ASTM). 1998.

ASTM D 129. Standard Test Method for Sulfur in Petroleum Products (General High Pressure Decomposition Device Method). American Society for Testing and Materials (ASTM). 2011.

ASTM D 189. Standard Test Method for Conradson Carbon Residue of Petroleum Products. American Society for Testing and Materials (ASTM). 2014.

ASTM D 240. Standard Test Method For Heat Of Combustion Of Liquid Hydrocarbon Fuels By Bomb Calorimeter. American Society for Testing and Materials (ASTM). 2009.

ASTM D 287. Standard Test Method for API Gravity of Crude Petroleum and Petroleum Products (Hydrometer Method). American Society for Testing and Materials (ASTM). 2012. 
ASTM D 482. Standard Test Method for Ash from Petroleum Products. American Society for Testing and Materials (ASTM). 2012.

ASTM D 3904. Test Method for Oil From Oil Shale (Resource Evaluation by the Fischer Assay Procedure) (Withdrawn 1996). American Society for Testing and Materials (ASTM). 1996.

Demirbaş, A. (2002). Asphaltene yields from five types of fuels via different methods. Energy Conversion and Management, 43(8), 1091-1097. doi: 10.1016/S0196-8904(01)00085-1.

EIA. (2012). Total Petroleum and Other Liquids Production. US Energy Information Administration. Consultado el 20 de noviembre de 2014. http://www.eia.gov/countries/cab. cfm?fips=VE.

Espitalié, J., Laporte, J., Madec, M., Marquis, F., Leplat, P., Paulet, J., and Boutefeu, A. (1977). Rapid method for source rocks characrerysation and for determination of petroleum potential and degree of evolution. OGST - Revue d'IFP Energies nouvelles, 32(1), 23-42. doi: 10.2516/ ogst:1977002.

Gerner, N.V, Koné, M., Ross, M.S., Pereira, A., Ulrich, A.C., Martin, J.W., and Liess, M. (2017). Stream invertebrate community structure at Canadian oil sands development is linked to concentration of bitumen-derived contaminants. Science of the Total Environment, 575, 1005-1013. doi: 10.1016/j.scitotenv.2016.09.169.

He, L., Li, X., Wu, G., Lin, F., and Sui, H. (2013). Distribution of saturates, aromatics, resins, and asphaltenes fractions in the bituminous layer of Athabasca oil sands. Energy \& Fuels, 27(8), 4677-4683. doi: 10.1021/ef400965m.

Hong, P.K.A., Cha, Z., Zhao, X., Cheng, C., and Duyvesteyn, W. (2013). Extraction of bitumen from oil sands with hot water and pressure cycles. Fuel Processing Technology, 106, 460-467. doi: 10.1016/j.fuproc.2012.09.013.

Hunt, J. (1995). Petroleum geochemistry and geology. (2nd ed). New York: W.H. Freeman and company.
Jiménez, L., y Cruz, M. (2004). Caracterización $y$ evaluación de reservas del yacimiento de asfaltitas en el sector de Isaza, municipio de Victoria, departamento de Caldas, Colombia. Manizales: Universidad de Caldas.

Lechner, C.A., Liapis, A.I., and Findley, M.E. (1989). A pyrolysis process for tar sands. The Canadian Journal of Chemical Engineering, 67(1), 85-95. doi: 10.1002/cjce.5450670112.

Liu, Q., Cui, Z., and Etsell, T.H.(2006). Characterization of Athabasca oil sands froth treatment tailings for heavy mineral recovery. Fuel, 85(5-6), 807-814. doi: 10.1016/j.fuel.2005.08.032

Pettijohn, F., Potter, P., and Siever, R. (1987). Sand and sandstone. (2nd. ed). New York: Verlag Springer.

Qing, W., Chunxia, J., Qianqian, J., Yin, W., and Wu, D. (2012). Combustion characteristics of Indonesian oil sands. Fuel Processing Technology, 99, 110114. doi: 10.1016/j.fuproc.2012.02.006.

Reyes-Caballero, F., Fernández-Morales, F.H., y Duarte, J.E. (2016). Panorama energético. Revista de Investigación, Desarrollo e Innovación, 7(1), 151-163. doi: 10.19053/20278306. v7.n1.2016.5605.

Reyes, Í. (1984). Geología de la Región de Duitama - Sogamoso - Paz de Rio - Belencito. Sogamoso: Universidad Pedagógica y Tecnológica de Colombia.

Ruíz-Acero, J.C., Reyes-Ortiz, O.J., y MorenoAnselmi, L.A. (2016). Evaluación del comportamiento mecánico de asfalto natural a partir de muestras a temperatura ambiente provenientes de Caquetá, Colombia. Revista de Investigación, Desarrollo e Innovación, 6(2), 145-154. doi: 10.19053/20278306.3115.

Sandoval, J.A. (2007). Estudio geológico y caracterización de las asfaltitas de la vereda de Paloblanco en el municipio de Vélez Santander. Tesis, Universidad Pedagógica y Tecnológica de Colombia.

Sherval, M. (2015). Canada's oil sands: The mark of a new "oil age" or a potential threat to Arctic 
security?. The Extractive Industries and Society, 2(2), 225-236. doi: 10.1016/j.exis.2015.01.011.

Sleep, S., McKellar, J.M., Bergerson, J.A., and MacLean, H.L. (2017). Expert assessments of emerging oil sands technologies. Journal of Cleaner Production, 144, 90-99. doi: 10.1016/j. jclepro.2016.12.107.

Sparks, B.D., Kotlyar, L.S., O'Carroll, J.B., and Chung, K.H. (2003). Athabasca oil sands: Effect of organic coated solids on bitumen recovery and quality. Journal of Petroleum Science and Engineering, 39(3-4), 417-430. doi: 10.1016/ S0920-4105(03)00080-9.

Speight, J.G. (2016). Introduction to enhanced recovery methods for heavy oil and tar sands. (2nd. ed). Boston: Gulf Professional Publishing.
Olga Patricia Gómez-Rojas

ORCID: 0000-0002-8382-929X

Ángeles G.-Borrego

ORCID: 0000-0001-9021-7358

Carlos Perea-Solano

ORCID: 0000-0003-0884-7938

Oswaldo Alfonso Sánchez-Morales

ORCID: 0000-0003-1068-7726

Hither Fernando Flórez-Molano

ORCID: 0000-0003-1627-3989

Trabajo recibido: agosto 8 de 2017

Trabajo aceptado: marzo 7 de 2018 